TOKYo J. MATH.

VoL. 15, No. 1, 1992

\title{
Unknotting Operations of Polygonal Type
}

\author{
Haruko AIDA \\ Tsuda College \\ (Communicated by K. Katayama)
}

\section{Introduction.}

In this paper, we consider about knots and links in the 3 -sphere $S^{\mathbf{3}}$.

A 3-gon move is a local move on a link diagram as indicated in Figure 1.1. In [3], Y. Nakanishi showed that a $\Delta$-unknotting operation can be realized by a finite sequence of 3-gon moves. A $\Delta$-unknotting operation is a local move on a diagram as shown in Figure 1.2, and it is a kind of unknotting operation ([2]). Hence a 3-gon move is a kind of unknotting operation. We generalize the notion of 3 -gon moves to $n$-gon moves as shown in Figure 1.3. In section 2, we obtain some results about $n$-gon moves which are similar to those about $\mathrm{H}(n)$-moves in [1]. For example, we will show that for given any knot $K$, there exists an integer $n$ such that $K$ can be transformed into a trivial knot by one $n$-gon move.

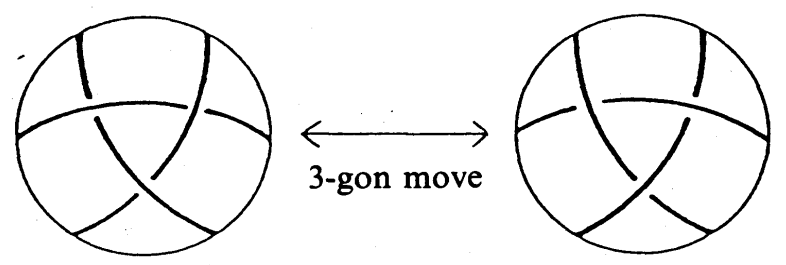

Figure 1.1.
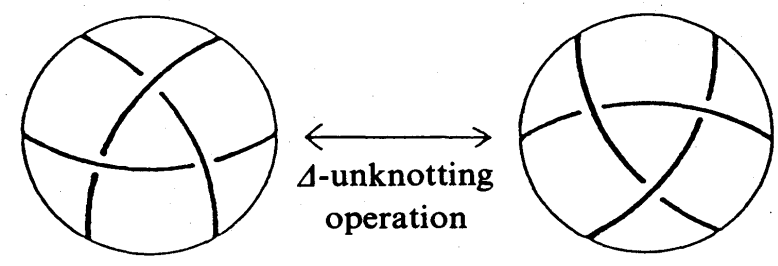

Figure 1.2. 

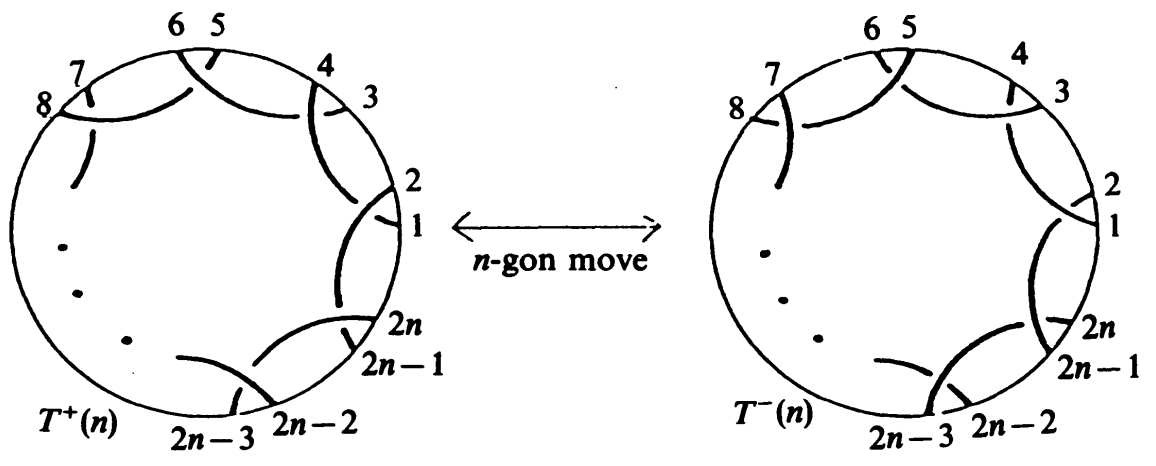

FIGURE 1.3.

In section 3, we will apply $n$-gon moves to links and will consider the number of the equivalence classes generated by $\boldsymbol{n}$-gon moves.

\section{2. n-gon moves on knots.}

DEFINITION. For any integer $n(\geq 3)$, an $n$-gon move on a link diagram is a local move on the diagram as indicated in Figure 1.3. And more precisely, under the notations $T^{+}(n), T^{-}(n)$ in Figure 1.3, an $n^{+}$-gon move on a diagram is a local move that replace a tangle $T^{+}(n)$ with a tangle $T^{-}(n)$ and an $n^{-}$-gon move on a diagram is the inverse move of an $n^{+}$-gon move.

REMARK. In fact, for any integer $n(\geq 3), n^{+}$-gon and $n^{-}$-gon moves are equivalent moves, i.e. each move can be realized by a finite sequence of the other moves.

DEFINITION. For any integer $n(\geq 3)$, an $n$-gon move on a link is a deformation of the link which corresponds to an $n$-gon move on a diagram of the link. Similarly, $n^{+}-g o n$ and $n^{-}$-gon moves on a link are defined.

Proposition 2.1. For any integer $n(\geq 3)$, every knot can be transformed into a trivial knot by a finite sequence of n-gon moves, i.e. an n-gon move is a kind of unknotting operation.

Proof. This follows from the following lemma:

LEMMA 1. (1) Any knot can be transformed into a trivial knot by a finite sequence of 3-gon moves.

(2) For any integer $n(\geq 3)$, an $n$-gon move can be realized by an $(n+1)$-gon move.

Proof. (1) In [3], Y. Nakanishi showed that a $\Delta$-unknotting operation can be realized by a finite sequence of 3-gon moves. So the proof is completed.

(2) Figure 2.1 illustrates how this can be accomplished. 

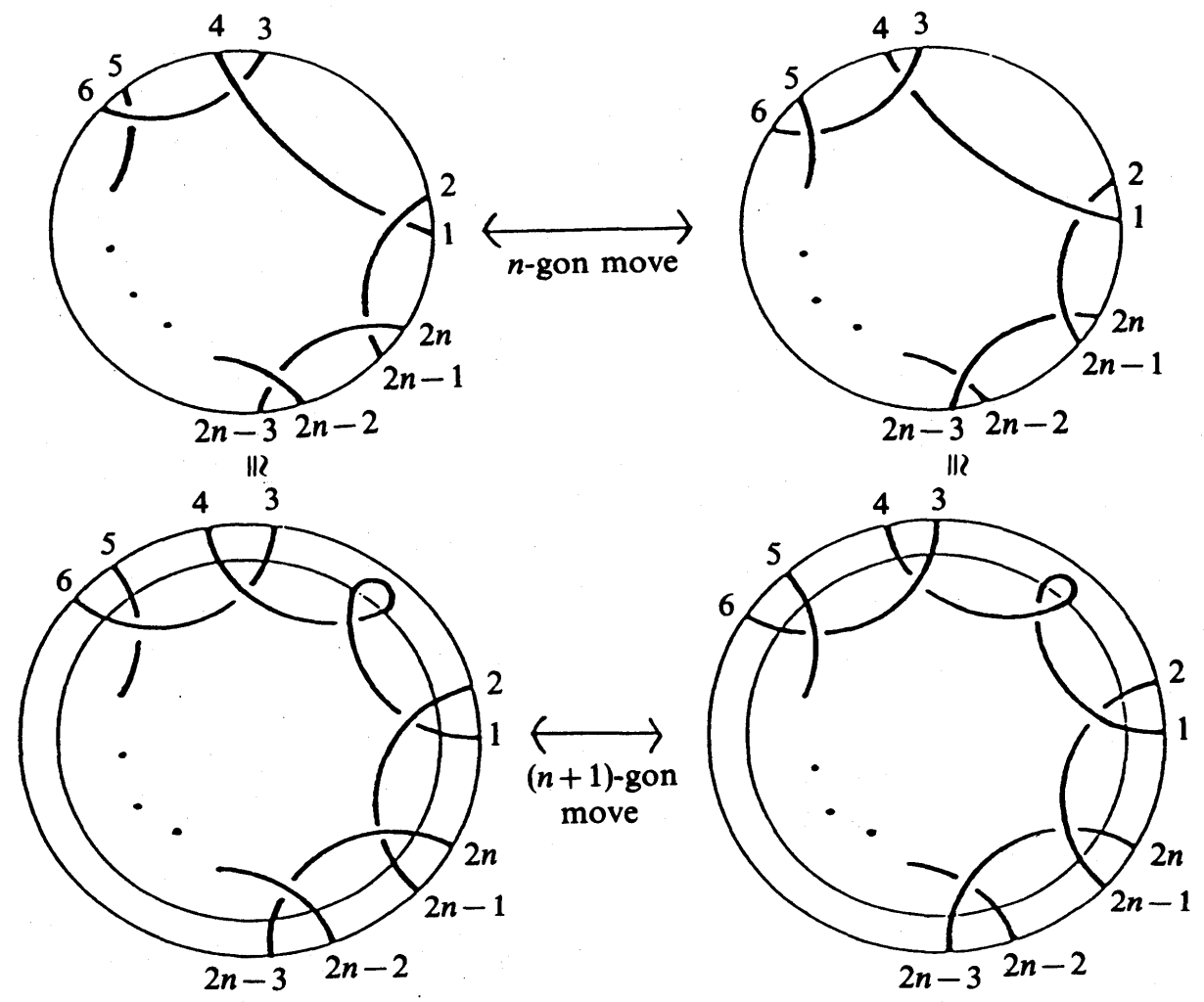

FIGURE 2.1.

Proposition 2.2. For any integer $n(\geq 3)$, every knot can be transformed into a trivial knot by a finite sequence of $n^{+}$-gon moves, i.e. an $n^{+}$-gon move is a kind of unknotting operation.

Proof. This follows from the following lemma:

LEMMA 2. (1) Any knot can be transformed into a trivial knot by a finite sequence of $3^{+}$-gon moves.

(2) For any integer $n(\geq 3)$, an $n^{+}$-gon move can be realized by an $(n+1)^{+}$-gon move.

Proof. (1) Figure 2.2 shows that a $\Delta$-unknotting operation can be realized by a finite sequence of $3^{+}$-gon moves.

(2) Figure 2.1 illustrates how this can be done. 


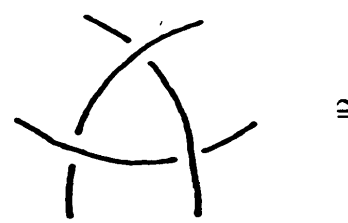

$\cong$
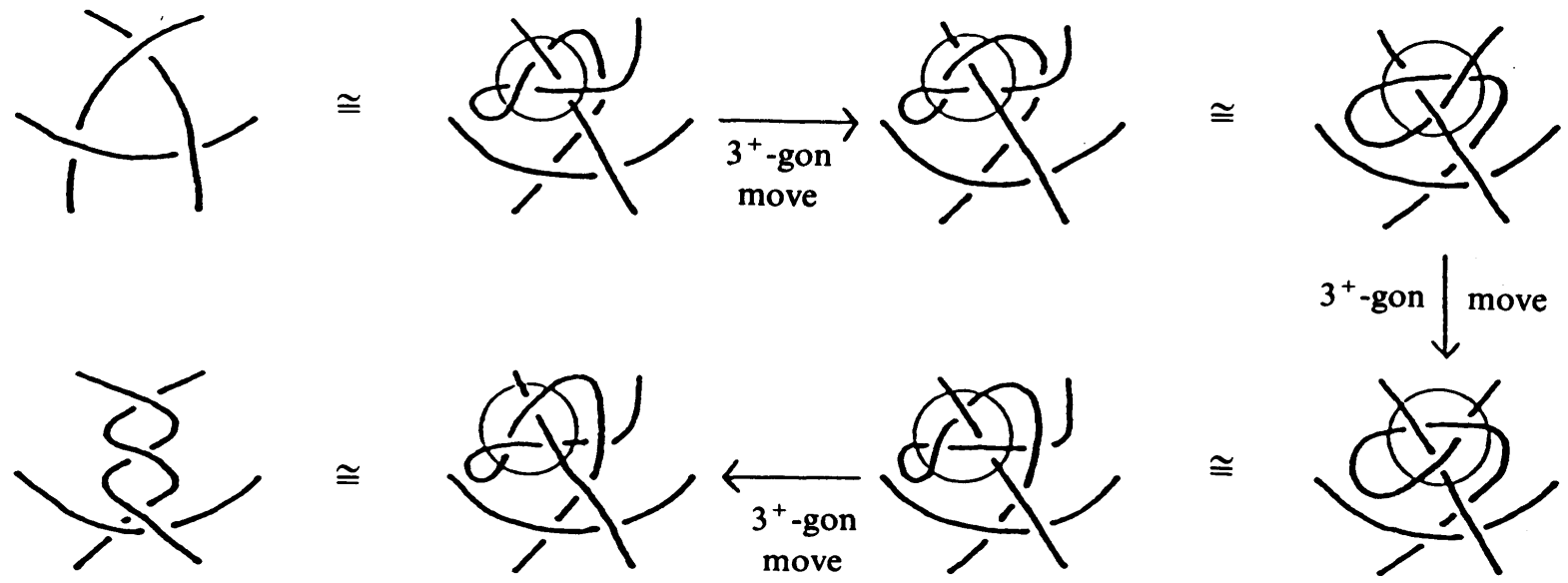

II)
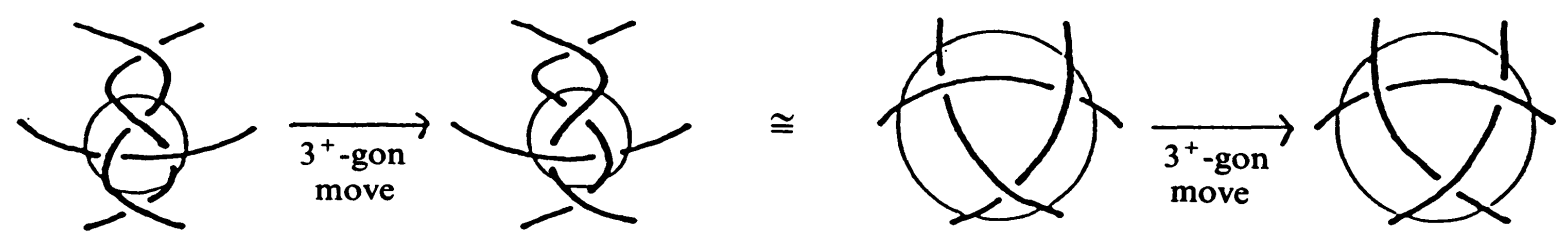

FIGURE 2.2.

REMARK. Similarly, we can also prove that an $n^{-}$-gon move is a kind of unknotting operation.

Definition. For a knot $K$ and an integer $n(\geq 3), u_{n}(K)$ is defined to be the minimum number of $n$-gon moves which are necessary to transform $K$ into a trivial knot.

For a knot $K$ and an integer $r(\geq 2)$, let $m g(K, r)$ denote the minimum number of generators of the first integral homology group of the $r$-fold cyclic branched covering space of $S^{3}$ branched along $K$.

Proposition 2.3. For a knot $K$ and integers $n(\geq 3)$ and $r(\geq 2)$, we have

$$
u_{n}(K) \geq m g(K, r) /(n-1)(r-1) .
$$

Proof. By replacing an $\mathbf{H}(n)$-move by an $n$-gon move in the proof of Theorem 4 in [1], we can obtain Proposition 2.3.

Proposition 2.4. For any knot $K$ and any integer $n(\geq 3)$, we have

$$
u_{n}(K) \geq u_{n+1}(K) \text {. }
$$

Proof. This is obvious from Lemma 1 (2).

THEOREM 2.5. For any knot $K$, there exists an integer $n$ such that $u_{n}(K)=1$.

PROOF. First of all, we show the following lemma: 
LEMMA 3. There exists a diagram $k$ of the knot $K$ satisfying the following condition:

(*) $k$ includes disjoint tangles $T_{1}^{+}(3), T_{2}^{+}(3), \cdots, T_{l}^{+}(3)$ for some $l$, so that, $k$ is transformed into a diagram of a trivial knot by $3^{+}$-gon moves on $T_{1}^{+}(3), T_{2}^{+}(3)$, $\cdots, T_{l}^{+}(3)$.

Proof of Lemma 3. By Proposition 2.2, $K$ can be transformed into a trivial knot by a finite sequence of $3^{+}$-gon moves. For each $3^{+}$-gon move in this sequence, attach a disk to $K$ as in Figure 2.3 such that these attaching disks are disjoint from each other. Thus $K$ is deformed into a trivial knot $K_{0}$ with attaching disks. Let $l$ be the number of these disks. Moreover, by contracting these disks, we may draw a diagram $k_{0}$ of $K_{0}$ with attaching disks, as follows:

$k_{0}$ contains disjoint 3-tangles $T S_{1}, T S_{2}, \cdots, T S_{l}$ with attaching disks as in Figure 2.4 , and the remainder part of $k_{0}$ is a diagram of disjoint arcs in $S^{3}$ (for example, see Figure 2.5).

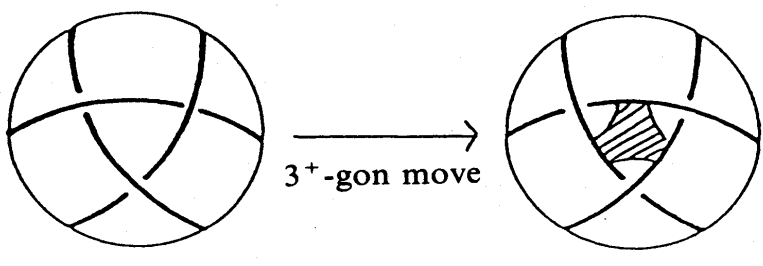

Figure 2.3.

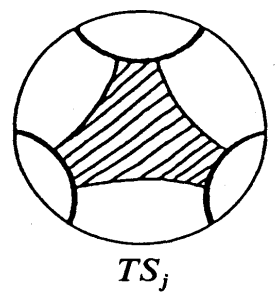

FIGURE 2.4 .

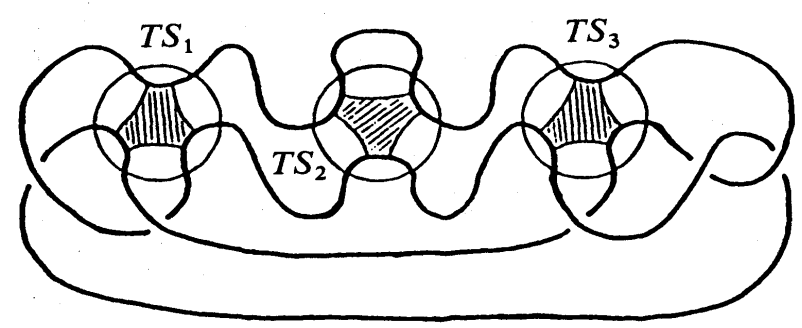

Figure 2.5 .

Finally we construct a diagram of $K$ in the following way. For each tangle $T S_{j}(j=1,2, \cdots, l)$ in $k_{0}$, we remove the attaching disk and apply a $3^{-}$-gon move as shown in Figure 2.6. Denote $T_{j}^{+}(3)$ be the resulting "small" 3-tangle in Figure 2.6. Then we obtain a diagram of $K$ which satisfies the condition (*).

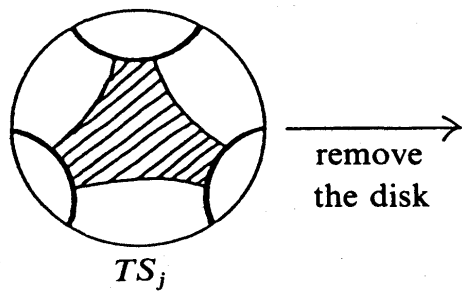

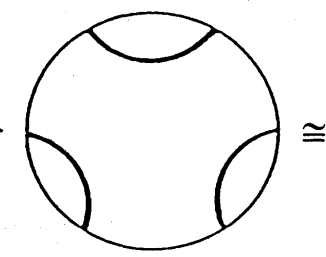

FIGURE 2.6.

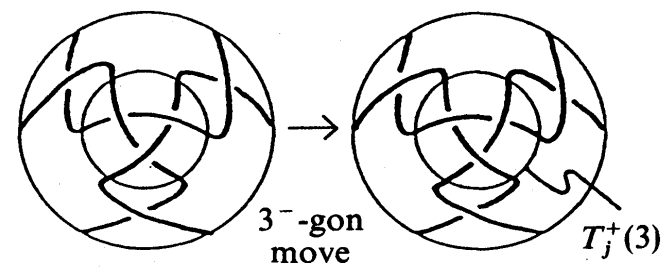


Let $k$ be a diagram of $K$ satisfying $\left({ }^{*}\right)$. More generally, we consider the following condition on a knot diagram $D$ :

$\left({ }^{* *}\right)_{l} \quad D$ includes $l$ disjoint tangles $T_{1}^{+}\left(n_{1}\right), T_{2}^{+}\left(n_{2}\right), \cdots, T_{l}^{+}\left(n_{l}\right)$ for some $l$ (where $n_{1}$, $\left.n_{2}, \cdots, n_{l} \geq 3\right)$, so that, by an $n_{i}^{+}$-gon move on $T_{i}^{+}\left(n_{i}\right)$ for all $i(1 \leq i \leq l), D$ is transformed into a diagram of a trivial knot.

Since $k$ satisfies $\left({ }^{* *}\right)_{l}$, so, by the induction on $l$, we can show Theorem 2.5 using the following lemma:

LemMa 4. Assume $l \geq 2$. Let $k$ be a diagram of $K$ satisfying $\left({ }^{* *}\right)_{l}$. Then there exists a diagram of $K$ satisfying $\left({ }^{* *}\right)_{l-1}$.

Proof of Lemma 4. Let $\boldsymbol{G}$ be the diagram which is obtained from $k$ by collapsing the tangles $T_{1}^{+}\left(n_{1}\right), T_{2}^{+}\left(n_{2}\right), \cdots, T_{l}^{+}\left(n_{l}\right)$ to vertices $v_{1}, v_{2}, \cdots, v_{l}$ respectively. Let $v_{i}$, $v_{j}$ be vertices which are connected by an edge $e$ in $G$.

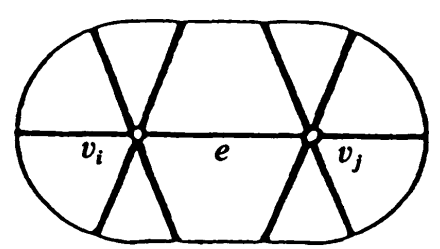

Figure 2.7.

For simplicity, we consider the case $n_{i}=n_{j}=3$. We can assume that there is a neighborhood $U$ of $v_{i}, v_{j}$ and $e$ in $S^{2}$ such that $U \cap G$ looks like Figure 2.7. If it is not, move $v_{j}$ along $e$ by an ambient isotopy of $S^{3}$, and then we can obtain a diagram as indicated in Figure 2.7. Therefore, we can assume that the corresponding part of $k$ looks like (a) or (b) in Figure 2.8. In the case (b), deform the diagram and choose the other tangle $T^{\prime+}(3)$ as shown in Figure 2.9. Then $3^{+}$-gon moves on $T_{i}^{+}(3)$ and $T_{j}^{+}(3)$ are equivalent to that on $T^{\prime+}(3)$. So we can reduce to the case Figure 2.8 (a).

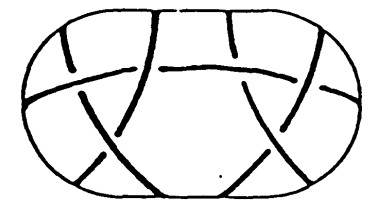

(a)

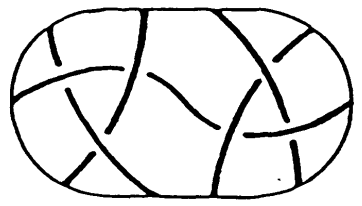

(b)

FIGURE 2.8 . 

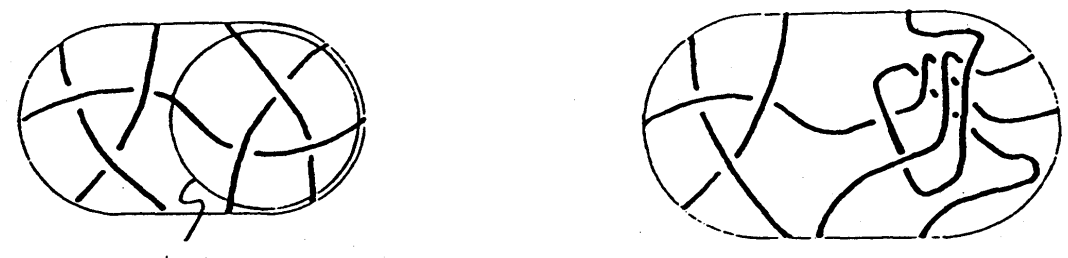

$T_{j}^{+}(3) \quad \sqrt{ }{ }^{2}$

\%

政
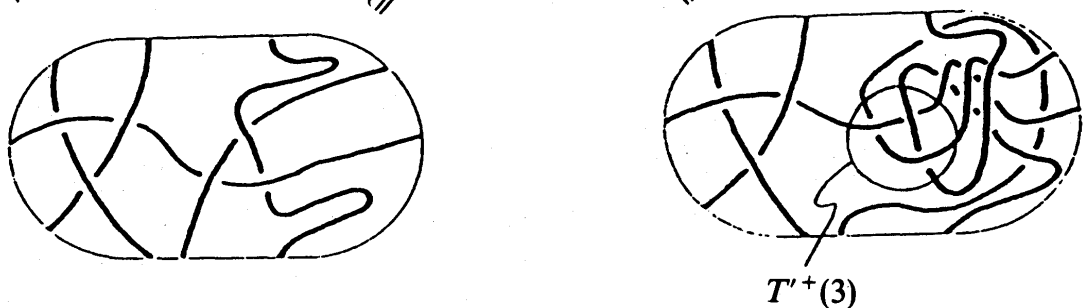

Figure 2.9.

Next deform the diagram $U \cap k$ and choose a tangle $T^{+}(3+3+2)=T^{+}(8)$ as shown in Figure 2.10. Then the $3^{+}$-gon moves on $T_{i}^{+}(3)$ and $T_{j}^{+}(3)$ in $k$ can be realized by an $8^{+}$-gon move on $T^{+}(8)$ in the new diagram. Hence the proof is completed in the case of $n_{i}=n_{j}=3$.

In the general case, by the same method, we can show that:

Two polygonal moves in $k$ (that is, the $n_{i}^{+}$-gon move on $T_{i}^{+}\left(n_{i}\right)$ and the $n_{j}^{+}$-gon move on $\left.T_{j}^{+}\left(n_{j}\right)\right)$ can be realized by a single $\left(n_{i}+n_{j}+2\right)$-gon move on $T^{+}\left(n_{i}+n_{j}+2\right)$ in another diagram of $K$.

COROLlaRY 2.6. For any knot $K$, we have

$$
\lim _{n \rightarrow \infty} u_{n}(K)=1 \text {. }
$$

Proof. This follows from Proposition 2.4 and Theorem 2.5.

DEFINITION. For a knot $K, p(K)$ is defined to be the minimum integer $n$ satisfying $u_{n}(K)=1$.

Proposition 2.7. For a knot $K$ and integers $n(\geq 3)$ and $r(\geq 2)$, we have

$$
p(K) \geq m g(K, r) /(r-1)+1 \text {. }
$$

Proof. This follows by letting $n=p(K)$ in Proposition 2.3.

EXAMPLE (Y. Nakanishi). Let $n$ be an integer which satisfies $n \geq 4$ and $n \not \equiv 0$ $(\bmod 3)$. Then the knot $K_{n}$ as indicated in Figure 2.11 has $p\left(K_{n}\right)=n($ where $n=3 k \pm 1)$.

This follows from Proposition 2.7 using $m g\left(K_{n}, 2\right)=n-1$.

\section{An equivalence relation of links.}

Let $L^{\mu}$ be the set of all $\mu$-component links. We define the equivalence relation $\tilde{n}$ 

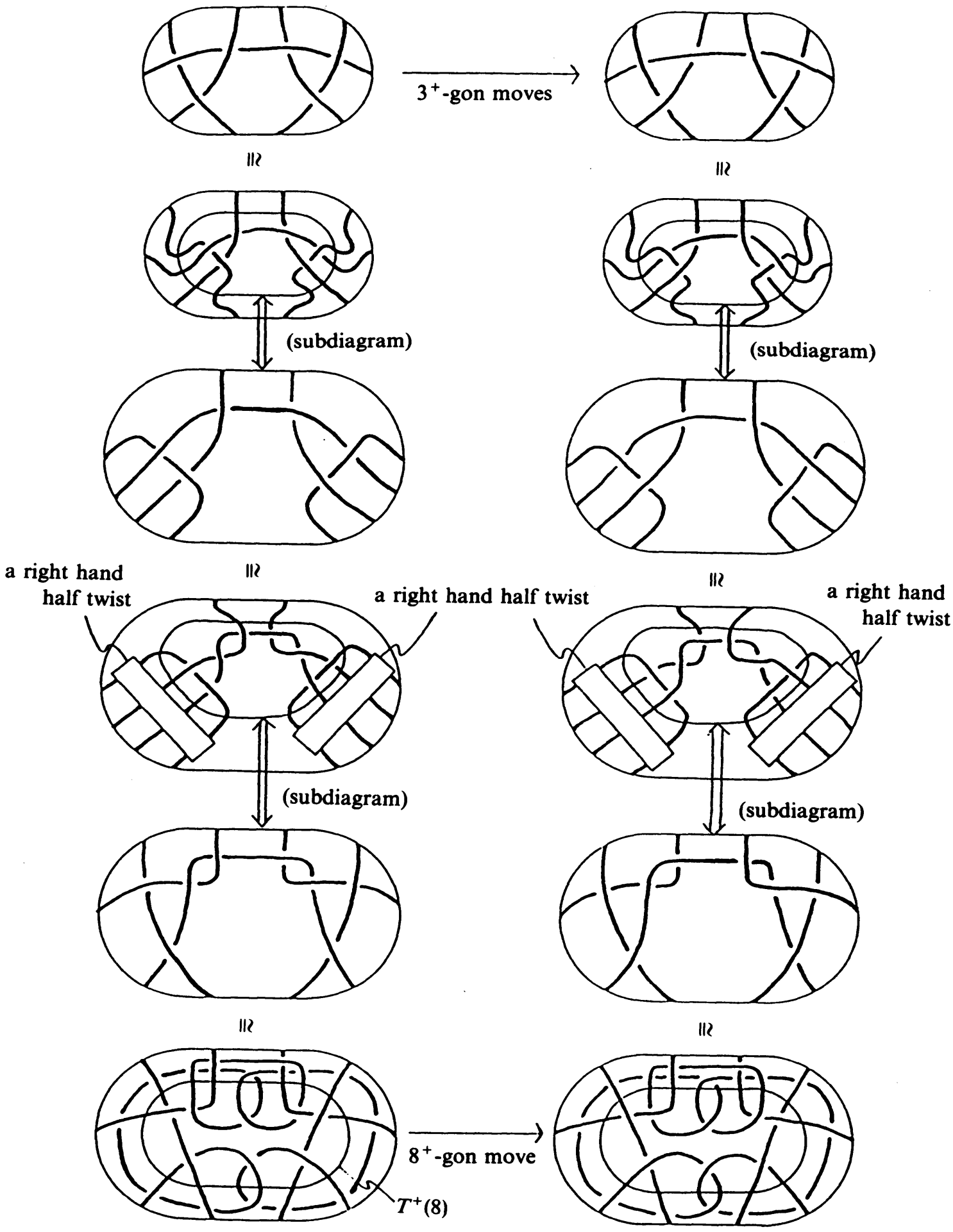

FIGURE 2.10. 


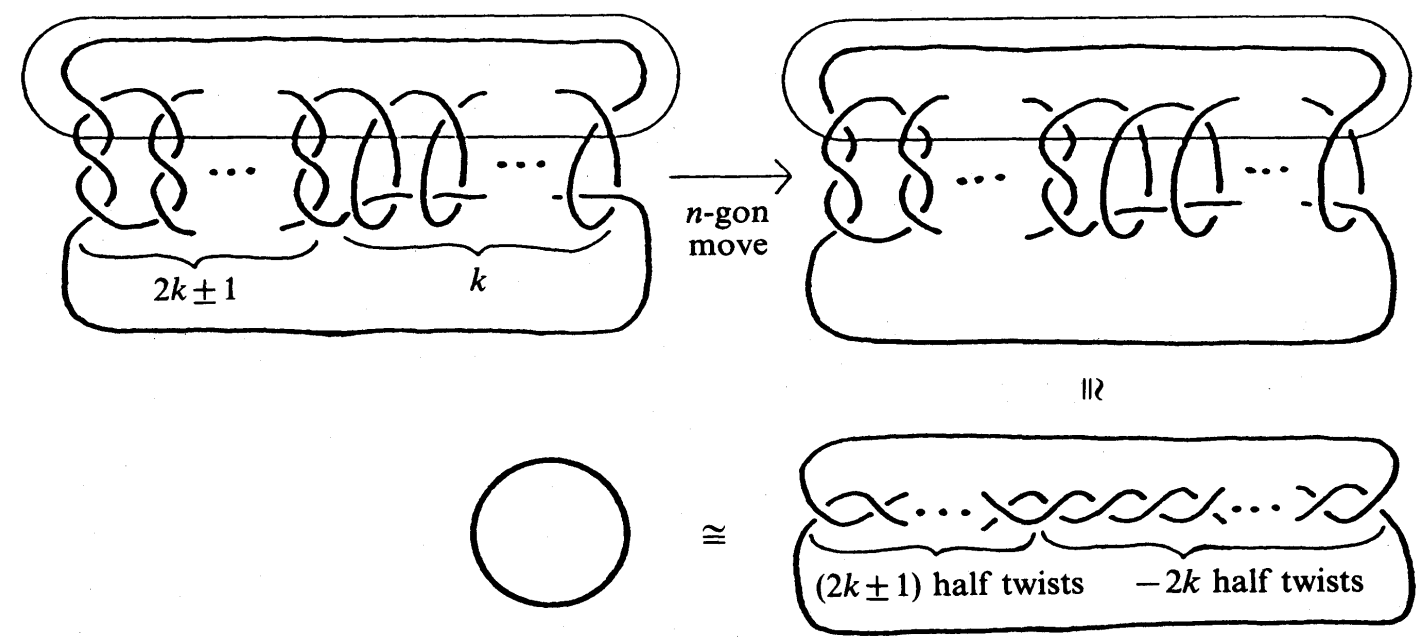

FIGURE 2.11.

for links $L, L^{\prime} \in L^{\mu}$ as follows:

$L_{\tilde{n}} L^{\prime} \underset{\text { def }}{\Longleftrightarrow} L$ is obtained from $L^{\prime}$ by a finite sequence of $n$-gon moves .

THEOREM 3.1. Let $L=K_{1} \cup K_{2} \cup \cdots \cup K_{\mu}$ and $L^{\prime}=K_{1}^{\prime} \cup K_{2}^{\prime} \cup \cdots \cup K_{\mu}^{\prime}$ be two $\mu$-component links. For any integer $n(\geq 3), L_{\widetilde{n}} L^{\prime}$ if and only if, after suitably oriented and/or ordered if necessary, $L$ and $L^{\prime}$ satisfy the following condition with respect to their linking numbers:

$$
\sum_{\substack{j=1 \\ j \neq i}}^{\mu} l k\left(K_{i}, K_{j}\right) \equiv \sum_{\substack{j=1 \\ j \neq i}}^{\mu} l k\left(K_{i}^{\prime}, K_{j}^{\prime}\right)(\bmod 2) \quad \text { for all } i=1,2, \cdots, \mu .
$$

Proof. At first, we prove the following lemma:

LEMMA 5. For any integer $n(\geq 4)$, an $n$-gon move can be realized by a finite sequence of $(n-1)$-gon moves.

Proof. This follows from Lemma 1 (2) and Figure 3.1.

By Lemma 1 (2) and 5, for any integers $m$ and $n(\geq 3)$, if $L_{\widetilde{m}} L^{\prime}$ then $L_{\widetilde{n}} L^{\prime}$. On the other hand, in [3], it was shown the case of $n=3$. So the proof is completed.

$L^{\mu} / \tilde{n}$ denotes the set of equivalence classes of $L^{\mu}$ by $\tilde{n}$.

COROLlaRY 3.2. For integers $\mu(\geq 1)$ and $n(\geq 3)$, we have

$$
\#\left(L^{\mu} / \widetilde{n}\right)=2^{\mu-1} \text {, }
$$

where $\# A$ denotes the number of the elements contained in $A$.

Proof. By Lemma 1 (2) and 5, the number of the equivalence classes generated by $n$-gon moves is independent of $n$. On the other hand, in [3], it was shown that 


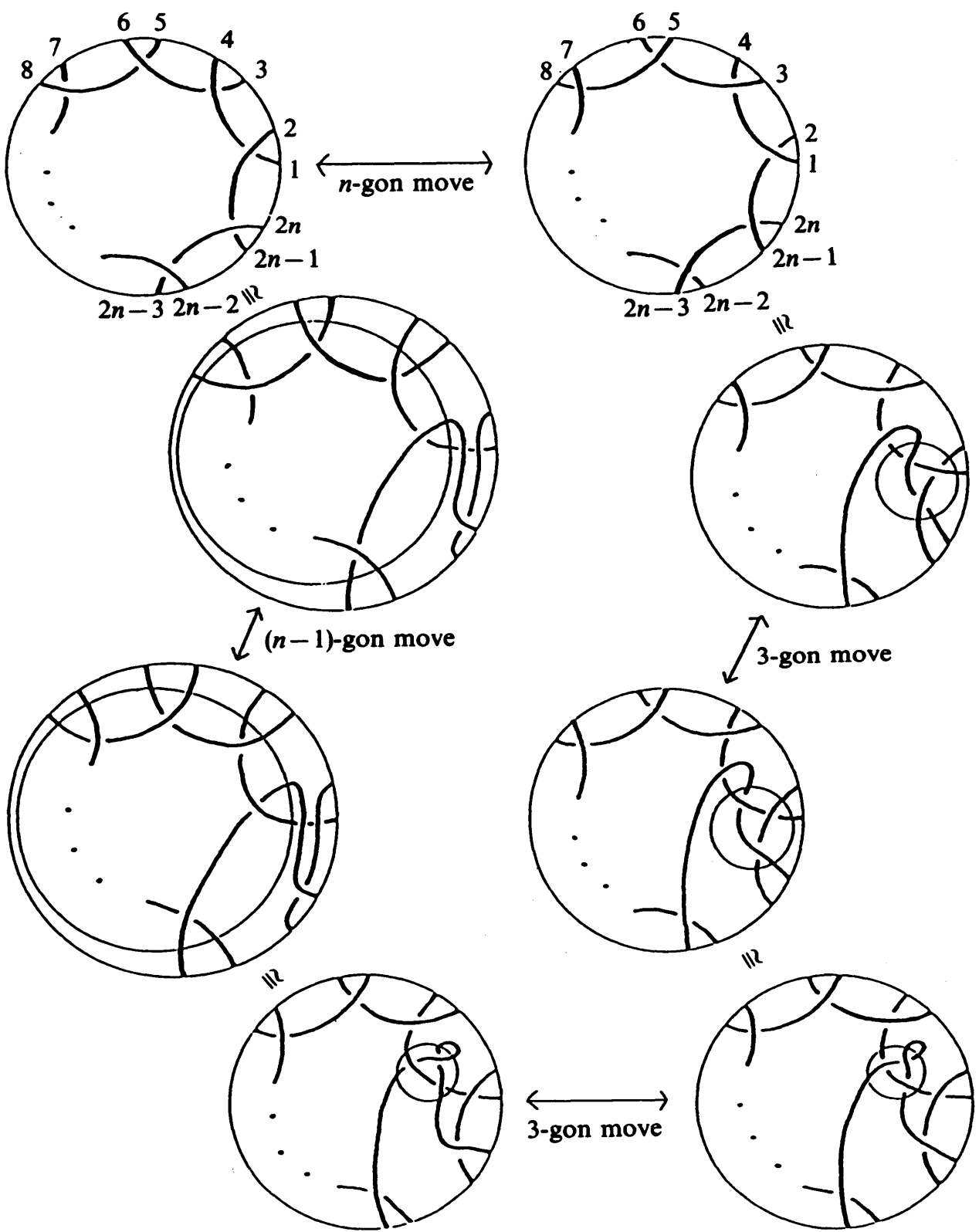

FigURe 3.1.

$\#\left(L^{\mu} / \widetilde{3}\right)=2^{\mu-1}$.

ACKnOwledgements. The author wishes to thank Professor K. Sakamoto, Professor Y. Nakanishi and Professor Y. Ohyama for their useful advices and encouragements. 


\section{References}

[ 1] J. Hoste, Y. Nakanishi and K. TaniYama, Unknotting operations involving trivial tangles, Osaka J. Math., 27 (1990), 555-566.

[2] H. MURAKami and Y. NAKANISHI, On a certain move generating link-homology, Math. Ann., 284 (1989), 75-89.

[ 3 ] Y. Nakanishi, Replacements in the Conway third identity, Tokyo J. Math., 14 (1991), 197-203.

Present Address:

Department of Mathematics, Tsuda College

TSUDA-MACHI, KODAIRA-SHI, TOKYO 187, JAPAN 